\title{
Learning Linear Constraints in Inductive Logic Programming
}

\author{
Lionel Martin and Christel Vrain \\ LIFO - Université d'Orléans - BP 6759 \\ 45067 Orléans Cedex 2 - France \\ email: $\{$ martin,cv $\} @$ lifo.univ-orleans.fr
}

\begin{abstract}
In this paper, we present a system, called ICC, that learns constrained logic programs containing function symbols. The particularity of our approach is to consider, as in the field of Constraint Logic Programming, a specific computation domain and to handle terms by taking into account their values in this domain. Nevertheless, an earlier version of our system was only able to learn constraints $X_{i}=t$, where $X_{i}$ is a variable and $t$ is a term. We propose here a method for learning linear constraints. It has already been a lot studied in the field of Statistical Learning Theory and for learning Oblic Decision Trees. As far as we know, the originality of our approach is to rely on a Linear Programming solver. Moreover, integrating it in ICC enables to learn non linear constraints.
\end{abstract}

\section{Introduction}

This paper is mainly devoted to the problem of handling numeric data in Inductive Logic Programming. This involves several points: defining a formal framework for studying this problem, learning numeric relations, introducing functional terms. From the semantic point of view, a consensus has emerged about the semantics of definite logic programs in terms of Herbrand interpretations, whereas several semantics have been defined for normal logic programs that can be based either upon the classical two-valued logics or upon multi-valued logics. Nevertheless, pure logic programs do not enable to express numeric expressions. In Prolog, meta-predicates, as for instance the primitive is $a$, have been defined to deal with numeric values, but the semantics of such programs can no longer be studied in terms of Herbrand interpretations. To deal with this problem, a new field, called Constraint Logic Programming, has rapidly grown: it enables to express constraints that are interpreted in a specific domain of computation, as for instance, the set of integers, or the reals.

In this paper, we propose a new approach for learning logic programs containing function symbols. Instead of relying on the syntactic form of terms, we propose to consider a domain of computation and to build new terms based on their values in this domain and on the interest of these values for discriminating positive and negative examples. In an earlier version [6], the prototype, called ICC, that we have developed in the framework of Constraint Logic Programming, 
was limited to constraints $X_{i}=t$, where $X_{i}$ is a variable and $t$ is a term in which $X_{i}$ does not occur. The main contribution of this paper is to propose a method, based on Linear Programming techniques, to learn relevant linear constraints and to integrate it in the system ICC, in order to learn non linear constraints.

This paper is organized as follows. Section 2 recalls basic definitions about Constraint Logic Programming. In Section 3, the system ICC is described. In Section 4, a method to learn linear inequalities is proposed as well as the way it is integrated in ICC.

\section{Basic definitions}

\subsection{Syntax}

We briefly recall some basic notions about constraint logic programming, that can be found in $[5,2]$. We consider:

- an infinite set of variables $\mathcal{V}$,

- a set, denoted by $\Sigma$, of function symbols,

- a set, denoted by $\Pi_{C}$, of constraint predicate symbols, containing at least the predicate $=$,

- a set, denoted by $\Pi_{P}$, of predicate symbols definable by a program.

A term over $\mathcal{V}$ and $\Sigma$ is inductively defined as follows: a variable $v$ of $\mathcal{V}$ is a term and, if $f$ is a function symbol of $\Sigma$, if $n$ is the arity of $f, n \geq 0$ and if $t_{1}, \ldots, t_{n}$ are terms then $f\left(t_{1}, \ldots, t_{n}\right)$ is a term.

A primitive constraint has the form $p\left(t_{1}, \ldots, t_{n}\right)$, where $p$ is a predicate symbol of $\Pi_{C}$ and $t_{1}, \ldots, t_{n}$ are terms.

A constraint is a first-order formula built with primitive constraints. In the remaining of the paper, we consider only the class $\mathcal{L}_{\Sigma}$ of constraints defined as the smallest set of constraints that contains all primitive constraints and is closed under variable renaming, conjunction and existential quantification.

An atom has the form $p\left(t_{1}, \ldots, t_{n}\right)$, where $p$ is a predicate symbol of $\Pi_{P}$ and $t_{1}, \ldots, t_{n}$ are terms.

A constrained atom is a pair $(c, p)$, where $c$ is a constraint and $p$ is an atom.

A constrained clause is an expression $a \leftarrow c \square b_{1}, \ldots, b_{n}$, where $c$ is a constraint and $b_{1}, \ldots b_{n}$ are atoms.

A CLP program, also called a constrained program, is a collection of constrained clauses.

Example 1. The following CLP program:

$$
\begin{aligned}
& \text { facto }(X, Y) \leftarrow X=0, Y=s(0) . \\
& \text { facto }(X, Y) \leftarrow X=s(Z), Y=T * X \square \text { facto }(Z, T) .
\end{aligned}
$$

defines factorial $\left(\Sigma=\{0, s, *\}, \Pi_{C}=\{=\}\right.$ and $\Pi_{P}=\{$ facto $\left.\}\right)$.

The variable $X$ is linked in the constrained clause $A_{0} \leftarrow c_{1}, \ldots, c_{m} \square A_{1}, \ldots, A_{n}$, if $X$ occurs in $A_{0}$ or, if there is a primitive constraint $c_{i}$ or an atom $A_{j}$ that contains the variables $X$ and $Y$ such that $Y$ is linked in $C$. A constrained clause $C$ is linked if all its variables are linked. 


\subsection{The constraint domain and its semantics}

In the field of Constraint Logic Programming, we usually consider a specific constraint domain over which computation is performed. A $\left(\Sigma, \Pi_{C}\right)$-structure $\mathcal{D}$ is composed of a non-empty set $D$, an assignment of a function $f_{\mathcal{D}}: D^{n} \rightarrow D$, for each $f \in \Sigma$, and an assignment of a function $p_{\mathcal{D}}: D^{n} \rightarrow\{$ True, False $\}$, for each $p \in \Pi_{C}$.

Example 2. In Example 1, a $\left(\Sigma, \Pi_{C}\right)$-structure can be defined on the domain $D$ of positive integers. It interprets the function 0 by the positive integer 0 , the function $s$ as the usual function successor on the set of positive integers, the function $*$ as the usual multiplication on the set of positive integers.

If $\mathcal{D}$ is a $\left(\Sigma, I I_{C}\right)$-structure, a $\mathcal{D}$-atom has the form $p\left(d_{1}, \ldots, d_{n}\right)$, where $p \in \Pi_{P}$ and $d_{i} \in D, 1 \leq 1 \leq n$. The set of all $\mathcal{D}$-atoms is called the $\mathcal{D}$-base.

Let $\mathcal{D}$ be a $\left(\Sigma, \Pi_{C}\right)$-structure and let $t$ be a ground term. The interpretation of the ground term $t$, w.r.t. $\mathcal{D}$, denoted by $I_{\mathcal{D}}(t)$, is defined as follows: if $f \in \Sigma$ and if $t_{1}, \ldots, t_{n}$ are terms, then $I_{\mathcal{D}}\left(f\left(t_{1}, \ldots, t_{n}\right)\right)=f_{\mathcal{D}}\left(I_{\mathcal{D}}\left(t_{1}\right), \ldots, I_{\mathcal{D}}\left(t_{n}\right)\right)$,

In the following, Const $(F)$ denotes the set of constants of $D$ appearing in the expression $F$ and $\operatorname{Term}_{\Sigma}\left(D^{\prime}\right)$ (where $D^{\prime}$ is a set of constants of $D$ ) denotes the set of terms built with constants of $D^{\prime}$ and function symbols of $\Sigma$.

If $\mathcal{D}$ is a $\left(\Sigma, \Pi_{C}\right)$-structure, a valuation $v$ is a function: $\mathcal{V} \rightarrow D$.

Let $v$ be a valuation, $v=\left\{X_{1} / d_{1}, \ldots, X_{n} / d_{n}\right\}$ where $d_{1}, \ldots, d_{n} \in D$. The set of inverse valuations of $v$, denoted by $V^{-1}(v)$, is defined by: $v^{-1} \in V^{-1}(v)$ iff $v^{-1}=\left\{d_{1} / X_{i_{1}}, \ldots, d_{n} / X_{i_{n}}\right\}$ with $v\left(X_{i_{j}}\right)=d_{j}$.

Let $A=p\left(d_{1}, \ldots, d_{n}\right)$ be a $\mathcal{D}$-atom and let $v$ be the valuation $\left\{X_{1} / d_{1}, \ldots, X_{n} / d_{n}\right\}$. If $v^{-1} \in V^{-1}(v), v^{-1}=\left\{d_{1} / X_{i_{1}}, \ldots, d_{n} / X_{i_{n}}\right\}$, then $v^{-1}(A)$ denotes the atoms $p\left(X_{i_{1}}, \ldots, X_{i_{n}}\right)$.

Let $\mathcal{D}$ be a $\left(\Sigma, \Pi_{C}\right)$-structure and let $T$ be a set of terms. An inverse interpretation of a constant $d$ w.r.t $T$ is the subset of $T$ composed of the terms $t$ that satisfy $I_{\mathcal{D}}(t)=d$. This set is denoted by $I_{T}^{-1}(d)$.

\section{The system ICC}

The learning task of ICC is specified by:

1. a set $\Sigma$ of function symbols and a set $\Pi_{C}$ of constraint predicates,

2. a $\left(\Sigma, \Pi_{C}\right)$-structure $\mathcal{D}$,

3. a set BASE of basic predicates defined by a set, denoted by $\mathrm{BK}^{+}$, of $\mathcal{D}$-atoms,

4. a set TARG of target predicates, specified by two sets of $\mathcal{D}$-atoms: $\mathrm{E}^{+}$and $\mathrm{E}^{-}$with $\mathrm{E}^{+} \cap \mathrm{E}^{-}=\emptyset$. The set $\mathrm{E}=\mathrm{E}^{+} \mathrm{U} \neg \mathrm{E}^{-}$represents the intended interpretation and in the following, $\Pi_{P}$ denotes the set BASE $\cup$ TARG. 
A $\mathcal{D}$-atom $e$ is $\mathcal{D}$-covered with respect to $\mathrm{E}^{+} \cup \mathrm{BK}^{+}$by a constrained clause $\mathrm{C}$ $=A_{0} \leftarrow c \square A_{1}, \ldots, A_{n}$ iff there exists a valuation $v$ which satisfies $c$ and such that $v\left(A_{0}\right)=e$ and $v\left(A_{i}\right) \in \mathrm{E}^{+} \cup \mathrm{BK}^{+}$for $i=1 \ldots n$. Such a valuation is called a covering valuation for $e$.

The aim of ICC is to find a constrained program, built over $\Sigma, \Pi_{C}$, and $\Pi_{P}$ which $\mathcal{D}$-covers the positive examples and which $\mathcal{D}$-covers no negative ones.

The method used to build linked constrained clauses is a classical one, that consists in iteratively adding to the body of the clause, either a constrained atom or a constraint until no negative example is $\mathcal{D}$-covered. We recall here the way relevant constraints are built. A similar method has been developed for building relevant constrained atoms. For more details, see [6].

Since a clause must $\mathcal{D}$-covers at least a $\mathcal{D}$-uncovered positive example, the first step of the algorithm is to choose (randomly) a $\mathcal{D}$-uncovered positive example $e \in \mathrm{E}^{+}$and to build a clause which $\mathcal{D}$-covers $e$ and as many $\mathcal{D}$-uncovered positive examples as possible. The positive example $e$ enables us to build a set of relevant constraints and a set of relevant constrained atoms, and then a classical entropy measure [10] enables to choose the best constraint or the best constrained atoms. Therefore, let $e \in \mathrm{E}^{+}$be a $\mathcal{D}$-uncovered positive example, let $C$ be a clause that $\mathcal{D}$-covers $e$ and some negative examples, and let $v$ be a covering valuation for $e$. The set of primitive constraints, denoted by Constr, that can be added to $C$ in order to refine it, is computed as follows:

Algorithm 1. Main Algorithm of ICC.

1- Compute the set $T=\operatorname{Term}_{\Sigma}($ Const $(v))$.

2- Compute $D^{\prime}=\left\{I_{\mathcal{D}}(t) \mid t \in T\right\}$.

3- Build $P C=\left\{p_{i}\left(d_{i_{1}}, \ldots, d_{i_{n}}\right)\right\} \mid p_{i} \in \Pi_{C}, d_{i_{j}} \in D^{\prime}, p_{i}\left(d_{i_{1}}, \ldots, d_{i_{n}}\right)$ true in $\left.\mathcal{D}\right\}$.

4- Compute $I_{T}^{-1}(P C)=\left\{I_{T}^{-1}(c c) \mid c c \in P C\right\}$

5- Remove trivial constraints from $I_{T}^{-1}(P C)$

6- Compute Constr $=\bigcup_{v^{-1} \in V^{-1}(v)} v^{-1}\left(I_{T}^{-1}(P C)\right)$

Example 3. Biases are introduced in ICC to reduce the set $T$, which have enabled ICC to learn the following constrained logic programs, respectively defining factorial and member.

\begin{tabular}{|l|c|c|}
\hline \multicolumn{1}{|c|}{ Learned program } & $\mathrm{E}^{+}$ & $\mid \mathrm{E}^{-1}$ \\
\hline \hline facto $(X, Y) \leftarrow X=0, Y=\operatorname{succ}(0)$. & 7 & 6 \\
facto $(X, Y) \leftarrow Z=\operatorname{pred}(X), T=\operatorname{div}(Y, X) \square$ facto $(Z, T)$. & & \\
\hline member $(X, Y) \leftarrow X=$ head $(Y)$. & 12 & 6 \\
member $(X, Y) \leftarrow Z=X, T=\operatorname{bod}(Y) \square$ member $(Z, T)$. & & \\
\hline
\end{tabular}

As has been mentioned in the introductory section, the constraints learned by ICC have been limited to expressions $X_{i}=t$, where $X_{i}$ denotes a variable and $t$ a term. In Section 4, we present a method for learning linear inequalities and in Section 5, we explain how it is integrated in ICC. 


\section{Learning linear constraints}

Let us suppose that we have built the clause $b_{0} \leftarrow c \square b_{1}, \ldots b_{n}$ and let us call $X_{1}, \ldots, X_{n}$ the numeric variables that have been introduced in this clause. Our goal is to find a linear constraint:

$$
c^{\prime}: a_{0}+a_{1} * X_{1}+\ldots+a_{n} * X_{n} \leq 0
$$

such that the clause $b_{0} \leftarrow c, c^{\prime} \square b_{1}, \ldots b_{n} \mathcal{D}$-covers a maximum of positive examples and a minimum of negative examples. The problem $\mathcal{P}$ that must be solved can be, more generally, stated as follows:

Let $X_{1}, \ldots, X_{n}$ be $n$ numeric variables. Let $\left[v_{i}, l a b_{i}\right]_{1 \leq i \leq m}$ be $m$ examples where $v_{i}$ denotes a valuation: $\left\{X_{1}, \ldots, X_{n}\right\} \rightarrow D$ and $l a b_{i} \in\{+,-\}$, depending whether $v_{i}$ represents a positive example or a negative one.

Find $a_{0}, \ldots, a_{n}$ such that:

- the number of valuations $v_{i}$ that satisfy $l a b_{i}=+$ and $a_{0}+a_{1} * v_{i}\left(X_{1}\right)+\ldots+$ $a_{n} * v_{i}\left(X_{n}\right) \leq 0$ is maximal, and

- the number of valuations $v_{i}$ that satisfy $l a b_{i}=-$ and $a_{0}+a_{1} * v_{i}\left(X_{1}\right)+\ldots+$ $a_{n} * v_{i}\left(X_{n}\right)>0$ is maximal.

In the following, $\mathcal{I} n_{i}^{+}$denotes the inequality $a_{0}+\Sigma_{j=1}^{n} a_{j} * v_{i}\left(X_{j}\right) \leq 0$, whereas $\mathcal{I} n_{i}^{-}$denotes the inequality $a_{0}+\sum_{j=1}^{n} a_{j} * v_{i}\left(X_{j}\right)>0$. In these inequalities, $v_{i}\left(X_{j}\right)$ are numeric values whereas $a_{0}, \ldots, a_{n}$ become variables.

Example 4. Let us suppose that we have two variables: $X_{1}$ represents the radius of a circle and $X_{2}$ represents the length of a square. Let us consider the target concept, expressing the following relation between a circle and a square: "the square can be drawn inside the circle". The set of examples is defined by: $\{[1,3,3,+],[2,3,3,+],[3,3,3,+],[4,3,3,+],[5,3,3,+],[6,3,3,+]$, $[7,3,3,-],[8,3,3,-],[9,3,3,-],[10,3,3,-],[11,3,3,-],[12,3,3,-]\}$

where a tuple $\left[i, c_{1}, c_{2}, l\right]$ represents the $i$-th example $\left(v_{i}, l a b_{i}\right)$ with $v_{i}=\left\{X_{1} / c_{1}\right.$, $\left.X_{2} / c_{2}\right\}$ and $l a b_{i}=l$.

In the best case, we would like to find $a_{0}, a_{1}, a_{2}$ such that for each $i=1 . .6, \mathcal{I} n_{i}^{+}$ is satisfied (for example $\mathcal{I} n_{1}^{+}=a_{0}+a_{1} * 3+a_{2} * 3 \leq 0$ ) and for each $j=7 . .12$, $\mathcal{I} n_{j}^{-}$is satisfied (for example $\mathcal{I} n_{\overline{7}}^{-}=a_{0}+a_{1} * 5+a_{2} * 2>0$ ).

A solution to $\mathcal{I} n_{i}^{+} \cup \mathcal{I} n_{i}^{-}$would give the equation of an hyperplane that separates the positive examples from the negative ones. Nevertheless, in many cases, this set has no solutions and we only aim at maximizing the number of inequalities that are satisfied. We solve this problem by using linear programming techniques, that can be expressed as follows:

$$
\left\{\begin{array}{c}
L P_{g}: \text { maximize } \sum_{j=1}^{n} c_{j} x_{j} \\
\text { subject to } \\
\Sigma_{j=1}^{n} a_{i j} x_{j} \leq b_{i}, \text { for } i=1,2, \ldots, m \\
l_{j} \leq x_{j} \leq u_{j}, \text { for } j=1, \ldots, n \\
\text { where } x_{j} \text { is a real or an integer. }
\end{array}\right.
$$


The problem $\mathcal{P}$ differs from the general $L P_{g}$ problem for two reasons: first, our objective function is only to maximize the number of satisfied inequalities; secondly, strict inequalities appear in $\mathcal{I} n_{j}^{-}$.

Therefore, we replace the set $\left[\mathcal{I} n_{i}^{+}\right]_{\left\{i \mid l a b_{i}=+\right\}}$ by the following set $\left[\mathcal{I} n_{i}^{\prime \prime+}\right]_{\left\{i \mid l a b_{i}=+\right\}}$ :

$$
\mathcal{I} n_{i}^{\prime \prime}: a_{0}+\Sigma_{j=1}^{n} a_{j} * v_{i}\left(X_{j}\right) \leq \sigma_{i} * C,
$$

where $C$ denotes a fixed constant the value of which will be very high and where $\sigma_{i}$ takes either the value 0 or 1 . The underlying idea is that if the value of $C$ is high enough, the inequality $a_{0}+\Sigma_{j=1}^{n} a_{j} * v_{i}\left(X_{j}\right) \leq \sigma_{i} * C$ will be satisfied with $\sigma_{i}=1$.

In the same way we built the set $\left[\mathcal{I} n_{i}^{\prime \prime-}\right]_{\left\{i \mid l a b_{i}=-\right\}}$ containing the following inequalities:

$$
\mathcal{I} n_{i}^{\prime \prime-}: a_{0}+\sum_{j=1}^{n} a_{j} * v_{i}\left(X_{j}\right) \geq \epsilon-\sigma_{i} * C
$$

where $\epsilon$ is a sufficiently low value which allows to remove the strict inequalities. The set of inequalities $\left[\mathcal{I n}_{i}^{\prime \prime+}\right]_{\left\{i \mid l a b_{i}=+\right\}} \cup\left[\mathcal{I} n_{i}^{\prime \prime-}\right]_{\left\{i \mid l a b_{i}=-\right\}}$ contains $(n+1)+m$ variables, $a_{0}, \ldots, a_{n}, \sigma_{1}, \ldots, \sigma_{m}$. Let us recall that the number $n$ is the number of numeric variables that have been introduced in the clause, whereas $m$ is the number of positive and negative instances.

Minimizing $\sum_{i=1}^{m} \sigma_{i}$ enables to maximize the number of inequalities that are satisfied among $\left[\mathcal{I} n_{i}^{+}\right]_{\left\{i \mid l a b_{i}=+\right\}} \cup\left[\mathcal{I} n_{i}^{\prime-}\right]_{\left\{i \mid l a b_{i}=-\right\}}$.

The linear problem that is solved can thus be stated as follows:

$$
\left\{\begin{array}{l}
\text { minimize } \sum_{i=1}^{m} \sigma_{i} \\
\text { subject to } \\
a_{0}+\sum_{j=1}^{n} a_{j} * v_{i}\left(X_{j}\right) \leq \sigma_{i} * C, i=1, \ldots, m \text { and } l a b_{i}=+, \\
a_{0}+\Sigma_{j=1}^{n} a_{j} * v_{i}\left(X_{j}\right) \geq \epsilon-\sigma_{i} * C, i=1, \ldots, m \text { and } l a b_{i}=- \\
a_{j} \in D, \text { for all } j=0, \ldots, n, \\
\sigma_{i} \in\{0,1\}, \text { for all } i=1, \ldots, m .
\end{array}\right.
$$

\section{Integration in ICC}

As has already been mentioned, ICC is based on a top-down strategy: while negative examples are extensionally covered, the current clause is refined by adding a literal to its body. Let us suppose that the variables $X_{1}, \ldots, X_{n}$ have already been introduced in the clause. Each time a new numeric variable is introduced, the system ICC calls the system $l p_{-}$solve, developed by M.R.C.M. Berkelaar ${ }^{1}$. When $l p_{-}$solve succeeds in solving the associated linear problem, the values of $\sigma_{i}$ enables to compute the number of positive instances and the number of negative instances that are $\mathcal{D}$-covered by the learned inequality which in turn, enable to compute the gain of this linear constraint. The literal that is introduced in the body of the clause is chosen, according to an entropy measure, among:

- the set of relevant constraints built by ICC,

${ }^{1}$ Eindhoven University of Technology, Eindhoven, The Netherlands 
- the set of relevant constrained atoms built by ICC,

- if possible, the inequality $a_{0}+a_{1} * X_{j_{1}}+\ldots+a_{p} * X_{j_{p}} \leq 0$, which expresses a relation between the numeric variables $X_{j_{1}}, \ldots, X_{j_{p}}$ which have already been introduced in the clause.

In the system ICC, it is possible to introduce function symbols, as for instance the function square $: \mathbb{N} \rightarrow \mathbb{N}$. In this case, ICC searches for constraints $c^{\prime}$ : $a_{0}+\sum_{i=1}^{m} a_{i} * X_{i} \leq 0$, where the variables $X_{i}$ are either numeric variables which appear in the clause, or terms built with variables appearing in the clause.

Example 5. Let us consider, for instance, the concept contains $(O, R)$ meaning that the object $O$ can be drawn inside a circle of radius $R$ and let us consider the basic predicate square $(O, N)$ expressing that $O$ is a square and that its length is $N$. If the function $s q r$ (that computes the square of a number) is given, then ICC learns the following program:

contains $(a, b) \leftarrow$ square $(a, b)$.

contains $(a, b) \leftarrow$ square $(a, d), 0.92 . \operatorname{sqr}(\mathrm{b})-0.40 . \operatorname{sqr}(\mathrm{d})-5 \mathrm{~b}+1.91 . \mathrm{d}+5.82>0$.

\section{Conclusion}

The system ICC is a system that builds, if possible, a constrained logic program, that $\mathcal{D}$-covers the positive examples and $\mathcal{D}$-covers no negative examples. It has been implemented in Sicstus Prolog on a Sun 4.

The experiments that we have made have already given positive results. Nevertheless, even if the system lp_solve that we use is efficient, so that searching for linear inequalities does not seem very expensive, the search space in ICC is, in general, very large due to the introduction of new terms. Biases have been introduced, as for instance the limitation of the depths of terms. Nevertheless, they are fixed before the learning process and we would like to study more dynamic biases linked to the learning problem and specifying terms or atoms that seem relevant.

Comparison with other works: Some systems already deal with the problem of learning constrained logic programs, as for instance, [11, 8]. In [11], the system combines a version space strategy with a divide-and-conquer strategy and the constraints that are learned mainly express bounds for variables. The strategy developed in [8] is an extension of Golem [7] to handle numeric constraints.

The problem of learning linear inequalities has already been a lot studied in the literature. It has been studied from a theoretical point of view, in [12]. There are two main differences with the Support Vector machines, defined in [12]: first, we intend to minimize the number of misclassified examples whereas their aim is to minimize the empirical risk; secondly, our method relies on Linear Programming techniques which is, as far as we know, original. This has also been studied in the field of learning decision trees, as for instance [1, 9]: generally, the underlying systems start from an initial inequation and perturb the coefficients until 
the impurity measure reaches a local minimum; in $\mathrm{OC} 1$ [9], a non deterministic step enables to get out the local minimum.

Finally, the idea of changing the feature space in order to learn non linear constraints has already been introduced in [12] and in [4]. Nevertheless, in our framework the functions that can be introduced in the equations depends on the underlying Constraint Logic Programming language, whereas in [4], the set of functions is reduced to the product between variables and to the square of a variable.

\section{References}

1. Breiman, Friedman, Olshem, Stone, 1993. Classification of Regression Trees. Chapman \& Hall.

2. Bergère M., Ferrand G., Le Berre F., Malfon B., Tessier A., 1995. La programmation logique avec contraintes revisitée en termes d'arbres de preuve et de squelettes. Rapport de recherche 95-06, LIFO, université d'Orléans.

3. Chvátal V., 1983. Linear Programming. Freeman.

4. Ittner A., Schlosser M., 1996. Non-Linear Decision Trees - NDT. Proceedings of the 13th International Conference on Machine Learning (ICML 96), L. Saitta (Ed.), Bari, Italy.

5. Jaffar J., Maher M.J., 1994. Constraint Logic Programming: A Survey. Jal of Logic Programming, vol. 19/20, may/july 1994, pp. 503-581, Elsevier Science Publishing.

6. Martin L., Vrain Ch., 1996. Induction of contraint logic programs. Proceedings of the Algorithmic Learning Theory workshop, ALT 96, Sidney, Australia.

7. Muggleton S., Feng C., 1992. Efficient Induction of Logic Programs. Inductive Logic programming. The A.P.I.C. Series $\mathrm{N}^{\circ} 38, \mathrm{~S}$. Muggleton (Ed.), Academic Press. pp. 281-298.

8. Mizoguchi F., Ohwada H., 1995. An Inductive Logic Programming Approach to Constraint Acquisition for Constraint-based Problem Solving. Proc. of the 5th Intl Workshop on Inductive Logic Programming, L. De Raedt (Ed.), pp. 297-323.

9. Murthy S., Kaisf S., Salzberg S., Beigel R., 1993. OC1: Randomized Induction of Oblique Decision Trees. Proceedings of the 11th National Conference on AI, AAAI-93, Cambridge, MIT Press, pp. 323-327

10. Quinlan J.R., 1990. Learning Logical Definitions from Relations. Machine Learning Journal, Vol. 5, Kluwer Academic Publishers, pp. 239-266.

11. Rouveirol C., Sebag M., 1995. Constraint Inductive Logic Programming. Proc. of the Fifth International Workshop on Inductive Logic Programming, L. De Raedt (Ed.), Leuven, September 1995, pp. 181-198.

12. Vapnik V.N., 1995. The Nature of Statistical Learning Theory. Springer. 